Sánchez-Torné, I., Pérez-Suárez, M., García-Río, E. y Baena-Luna, P. (2021). ¿Cómo influye el género en la intención emprendedora? Un análisis del alumnado universitario. Revista de Investigación Educativa, 39(2), 427-444.

DOI: http://dx.doi.org/10.6018/rie.428451

\title{
¿Cómo influye el género en la intención emprendedora? Un análisis del alumnado universitario
}

\section{How does gender influence in entrepreneurial intention? An analysis of university students}

\begin{abstract}
Isadora Sánchez-Torné*, Macarena Pérez-Suárez*, Esther García-Río** y Pedro Baena-Luna**
*Departamento de Economía Aplicada III. Facultad de Ciencias Económicas y Empresariales. Universidad de Sevilla. ** Departamento de Administración de Empresas y Marketing. Facultad de Ciencias Económicas y Empresariales. Universidad de Sevilla.
\end{abstract}

\begin{abstract}
Resumen
Este trabajo determina qué elementos influyen en la potencial propensión a actuar (PA) a la hora de emprender desde una perspectiva de género del alumnado universitario. Se ha realizado a partir del Modelo del Evento Emprendedor de Shapero y Sokol (1982) y las investigaciones empíricas de Liñán y Chen (2009) sobre la modelización de variables para medir la PA con el fin de emprender. Se suministró un cuestionario al alumnado de último curso del Grado de Relaciones Laborales y Recursos Humanos de la Universidad de Sevilla, entre los años académicos 2012-2013 a 2019-2020, obteniéndose un total de 663 respuestas. Los resultados evidencian que existen similitudes y diferencias entre la PA del alumnado. En el caso de las variables que influyen en la PA de hombres y mujeres se encuentran la conveniencia percibida (CP), la percepción de viabilidad (PV) y el hecho de tener una persona progenitora emprendedora. Entre las que se identifican diferencias destacan, la deseabilidad de ser persona emprendedora y algunas capacidades específicas. Esta investigación pone de relieve la importancia de adaptar la educación emprendedora a los distintos perfiles de alumnado en una universidad.

Palabras clave: intención emprendedora; alumnado universitario; género; emprendimiento.
\end{abstract}

Correspondencia: Pedro Baena-Luna, pbaenaluna@us.es. Departamento de Administración de Empresas y Marketing, Universidad de Sevilla, Avd. Ramón y Cajal nº1, 41.018, Sevilla (España). 


\begin{abstract}
With its focus on gender, this work determines which elements influence the potential propensity to act (PA) when university students decide to start a business. It is based on Shapero and Sokol's Entrepreneurial Event Model (1982) and Liñán and Chen's empirical research (2009) on the variable modelling to measure the predisposition to start a business. A questionnaire was provided to the final year students of the Labor Relations and Human Resources Degree at the University of Seville, between the academic years 2012-2013 and 2019-2020, obtaining a total of 663 responses. The results obtained show that there are similarities and differences between the students' PA. In the case of the variables that influence the PA of men and women, we find the perceived convenience $(P C)$, the perception of viability $(P V)$ and the fact of having an entrepreneurial parent. Among the differences identified, the willingness of being an entrepreneur and some specific capabilities stand out. This research highlights the importance of adapting entrepreneurship education to the different student profiles at university.

Keywords: entrepreneurial intentions; university students; gender; entrepreneurship.
\end{abstract}

\title{
Introducción
}

En los últimos años las universidades no solo han desarrollado acciones en el ámbito educativo, han implementado también otro tipo de actuaciones dirigidas a mejorar la empleabilidad de las personas egresadas (Naval-Castelao, González-Pascual, JordánRamos y Ruiz-Pomada, 2015). Las universidades se implican de manera más activa y comprometida con la sociedad actual. Para ello promueven una mayor interacción con los actores económicos de los territorios (Schmitz, Urbano, Dandolini, de Souza y Guerrero, 2017) e incentivan la conversión de sus universidades en instituciones emprendedoras (Medina, Moncayo, Jácome y Albarrasin, 2017).

Los programas universitarios de educación emprendedora han sido analizados intensamente en la búsqueda de potenciales beneficios (Karimi, Biemans, Lans, Mulder y Chizari, 2012). Como principal objetivo tienen el favorecimiento de la adquisición de competencias y habilidades por parte del alumnado, de tal manera que les permita sentirse capacitados para crear sus propios puestos de trabajo a través de la opción del emprendimiento (Premand, Brodmann, Almeida, Grun y Barouni, 2016). La sociedad requiere también de un emprendimiento universitario, y para ello, es necesario tener a un alumnado preparado y entrenado en capacidades emprendedoras (CE) (Medina et al., 2017). Esta capacitación posibilita que el proceso que se inicia con la generación de una idea pueda llegar al mercado en forma de iniciativa emprendedora real (MartínezGarcía, Padilla-Carmona y Suárez-Ortega, 2019).

Ha de tenerse en cuenta que las acciones desarrolladas en estos programas de educación emprendedora puedan tener resultados distintos. Gielnik, Uy, Funken y Bischoff (2017) señalan, como, si bien se conoce que la educación emprendedora es eficaz a la hora de influir en la mentalidad emprendedora del alumnado gracias al desarrollo de intenciones emprendedoras (IE) (Asghar, Gul, Seitamaa-Hakkarainen y Tasdemir, 2019) 
no se dispone aún de un profundo conocimiento teórico sobre cuáles son los procesos dinámicos de esta capacitación que llevan a la creación de una empresa.

De forma paralela al auge en la puesta en marcha de programas de educación emprendedora, siendo una de las formas más utilizadas al analizar el fenómeno del emprendimiento, se encuentra el estudio de los modelos de intención emprendedora (González-Serrano et al., 2016). Estos trabajos ponen el foco en la valoración de las intenciones de las personas a la hora de poner en marcha una iniciativa de carácter emprendedor (Karimi et al., 2012). Investigaciones relacionadas constatan como, si bien la educación emprendedora no siempre se materializa en la puesta en marcha de un proyecto empresarial (Baena-Luna, García-Río y Monge-Agüero, 2020) sí que afecta positivamente en la IE de estas personas (Maresch, Harms, Kailer y WimmerWurm, 2016).

Junto al estudio de la potencial IE de distintos grupos y colectivos, en los últimos años es habitual la incorporación también de una perspectiva de género en el ámbito de la investigación en emprendimiento y en otras disciplinas. En el caso de la IE no hay un claro consenso respecto a las posibles diferencias y similitudes a la hora de emprender por parte de los hombres y de las mujeres (Lim y Envick, 2013). Sin embargo, esto no es óbice, para sí poder constatar cómo existe aún una clara brecha de género entre hombres y mujeres en cuanto a la potencial acción y capacidad emprendedora (Sánchez-Cañizares y Fuentes-García, 2013; Sánchez-Torné y Pérez-Suárez, 2019).

Aunque aún en la actualidad, algunos trabajos del área de la gestión empresarial establecen que no existen diferencias o barreras en el caso de los hombres frente a las mujeres a la hora de emprender, no es menos cierto que sigue existiendo un orden jerárquico de género donde la mujer es asociada a la potencial debilidad de sus acciones mientras que el hombre es vinculado a hacia un claro discurso emprendedor (Zambrano-Vargas y Vázquez-García, 2019). Este es un hecho que se encuentra no solo presente en el caso de la intención o la propensión a emprender, sino también, en las empresas de alto potencial de crecimiento. Aún en la actualidad es menor el porcentaje de mujeres fundadoras de proyectos empresariales de este tipo (Startiene y Remeikiene, 2008). Esta situación viene a corroborar como los estereotipos de género condicionan a los hombres y a las mujeres que tienen interés en poner en marcha una iniciativa emprendedora (Gupta, Wieland, y Turban, 2019).

En consecuencia, el objetivo de este trabajo es conocer los elementos significativos que afectan al potencial de la IE en el caso del alumnado universitario. Este análisis se ha realizado incorporando la perspectiva de género con el fin de poder evidenciar si estos elementos son diferentes en el caso de los hombres y de las mujeres. Un mejor conocimiento de estas realidades y sus relaciones va a permitir una mejora en la calidad de la educación emprendedora. Esto a su vez influirá de manera directa y positiva en la mejora de la potencial empleabilidad de las personas egresadas.

La estructura de este trabajo es la siguiente: comienza con esta introducción que antecede a los conceptos de intención emprendedora y género abordados en el marco teórico. Posteriormente, es mostrada la metodología seguida a la hora de acometer el objetivo de la investigación y obtenido en los resultados junto con una discusión propia. El trabajo finaliza exponiendo las conclusiones derivadas más notables. 


\section{Marco Teórico}

En el ámbito del emprendimiento distintos trabajos relacionados han venido a demostrar cómo, en el caso del comportamiento humano, las intenciones se han evidenciado como un fuerte descriptor (Karimi et al., 2012). Sin embargo, antes de que pueda haber una iniciativa emprendedora, debe existir una intención previa para el emprendimiento (Klapper y Léger-Jarniou, 2006). Es ahí, y en el caso del emprendimiento universitario, donde será oportuno observar el nivel de influencia que tiene la educación emprendedora (Maresch et al., 2016).

El estudio de la IE no es nuevo. Desde los años 80 del siglo XX ha habido distintos modelos teóricos que han abordado esta realidad. Los dos más con más relevancia y que han generado una mayor producción científica relacionada han sido los de Shapero y Sokol (1982) y el de Ajzen (1991). Ambos modelos tienen como denominador común el vínculo cognitivo entre los antecedentes de la IE, la propia IE y la acción emprendedora (Zhang, 2017). En particular, el modelo Shapero y Sokol (1982) representa una combinación de factores sociales y culturales (Cera, Mlouk, Cera y Shumeli, 2020). Este modelo pone de manifiesto que la intención para emprender se fundamenta a través de tres elementos: la conveniencia percibida $(\mathrm{CP})$, la propensión a actuar (PA) y la percepción de viabilidad (PV). La CP ha de ser entendida como el potencial atractivo que para una persona tiene el hecho de poner en marcha una iniciativa emprendedora. La PV se establece a partir de la propia confianza de la persona sobre su capacidad a la hora de comenzar una iniciativa de este tipo. Por último, la PA es la disposición de la persona a tener comportamientos decididos ante una posible oportunidad (Kuehn, 2008).

El modelo de Shapero y Sokol (1982) incide en identificar aquellos potenciales factores de carácter situacional, social e individual que pueden conducir a la persona hacia el emprendimiento. Parte del hecho de que la intención de una persona para poner en marcha una iniciativa emprendedora se ve influida tanto por las percepciones de esta respecto a su potencial de conveniencia y viabilidad, como de su actitud positiva a la hora de actuar ante la identificación de oportunidades (Klapper y Léger-Jarniou, 2006).

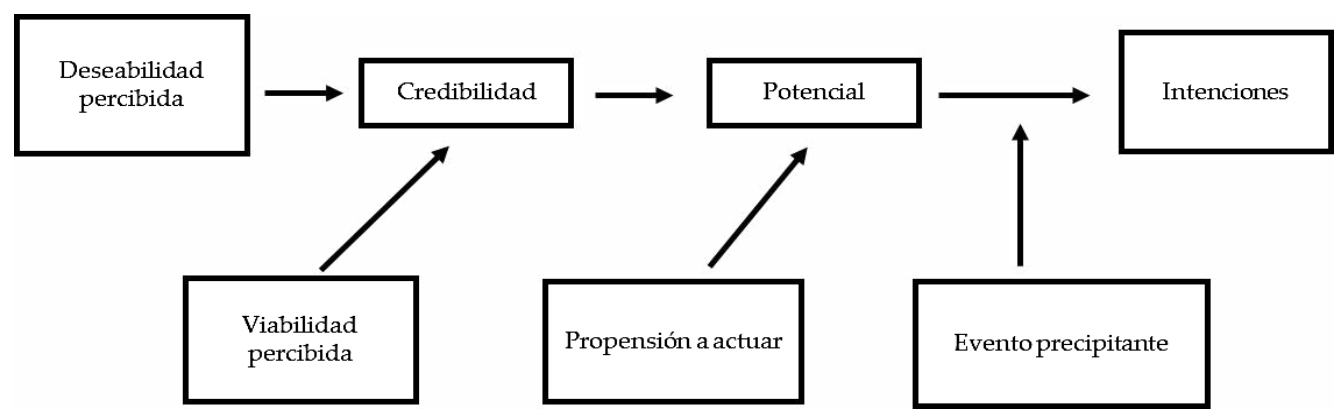

Figura 1. Modelo de Intención Emprendedora.

Fuente: Rai et al. (2017) adaptado de Shapero y Sokol (1982). 
A partir de los modelos de IE de Shapero y Sokol (1982) y el de Ajzen (1991), Krueger y Brazeal (1994) establecieron la equivalencia entre las variables que los componen y su significado (Tabla 1).

Tabla 1

Equivalencia entre las variables de las principales teorías

\begin{tabular}{ll}
\hline \multicolumn{1}{c}{$\begin{array}{c}\text { Variables de la Teoría del Comportamiento } \\
\text { Planificado de Ajzen }\end{array}$} & \multicolumn{1}{c}{$\begin{array}{c}\text { Variables del Modelo del Evento } \\
\text { Emprendedor de Shapero y Sokol }\end{array}$} \\
\hline Actitud ante el Emprendimiento (AE) & Conveniencia Percibida (CP) \\
Control del Comportamiento Percibido (CCP) & Percepción de Viabilidad (PV) \\
Intención Emprendedora (IE) & Propensión a Actuar (PA) \\
\hline
\end{tabular}

Fuente: Elaboración propia a partir de Krueger y Brazeal (1994).

Tradicionalmente se ha considerado que la actitud personal ante el emprendimiento ejerce una influencia sobre la potencial IE de las personas (Zhang, Duysters y Cloodt, 2014). La AE es entendida como aquello que la persona encuentra deseable y que es dependiente del probable impacto personal que tenga la realización del comportamiento objetivo. Sin embargo, no existe unanimidad acerca de una influencia directa de la CP sobre la PA. Algo similar ocurre en el caso de los trabajos que sugieren que la $\mathrm{CP}$ ha de ser vista como una influencia moderadora en lugar de un antecedente directo de la PA (Klapper y Léger-Jarniou, 2006). En definitiva, de una manera directa o indirecta, el alumnado hombre o mujer que tiene una actitud favorable hacia el emprendimiento es más propenso a convertirse en una persona emprendedora (Zhang et al., 2014). A partir de estas consideraciones respecto a la CP y su influencia en la PA puede establecerse la primera de las hipótesis de este trabajo.

$H_{1}$ : La Conveniencia Percibida (CP) influye en la Propensión a Actuar (PA) tanto de las alumnas como de los alumnos.

La PV es el grado en que la persona emprendedora potencial cree en ella misma. Es decir, si siente o no que es capaz de iniciar una iniciativa emprendedora. La PV puede depender de distintos elementos como señalan Klapper y Léger-Jarniou (2006). La relación entre la PV y la PA no siempre es positiva, incluso en el caso del alumnado universitario hombre y mujer (Zhang et al., 2014; Romero-Martínez y Milone, 2016). En la mayoría de los casos la persona establece como deseable crear una empresa, aunque su percepción de la potencial viabilidad no sea positiva. Por tanto, el CCP y la AE son cercanos a la PV y la CP (Autio, Keeley, Klofsten, Parker y Hay, 2001). El impacto de la CP y la PV se demuestran en la PA (Ali, Lu y Wang, 2012). Por tanto, no observándose diferencias en el caso de hombres y mujeres en este punto se puede establecer que:

$H_{2}$ : La Percepción de Viabilidad ( $P$ la Propensión a Actuar (PA) tanto de las alumnas como de los alumnos. 
En la PA pueden influir variables como la cultura, la familia, las personas compañeras, amigas y/o mentoras, así como, el apoyo de personas emprendedoras de éxito (Autio et al., 2001). Personas con alguna experiencia previa en empresas o negocios familiares pueden incorporar sus vivencias, pues su PV puede influir positivamente o de manera negativa en la creación de empresas (Garba, Kabir y Nalado, 2014). Por tanto, la PA es "influenciada por diferentes factores externos, que pueden actuar sobre la motivación, las cualidades personales o las necesidades percibidas para emprender. Esas influencias son la trayectoria vital de cada estudiante" (Naval-Castelao et al., 2015, p.200). Sin embargo, en el caso particular de las mujeres estudiantes universitarias, esta influencia se muestra en menor medida que en el caso de los hombres estudiantes universitarios (Neira, Portela, Fernández y Rodeiro, 2013). Esto da pie a la tercera hipótesis de este trabajo, que relaciona los modelos de referencia emprendedora con la PA y el género.

$H_{3}$ : Los modelos de referencia que tienen una relación positiva con la Propensión Actuar (PA) es distinta entre alumnas y alumnos.

Distintas capacidades humanas pueden tener diferente nivel de influencia en la PA a través de una relación positiva. Sin embargo, hay una relación negativa en el desarrollo de las capacidades relacionadas con la posibilidad de reconocer oportunidades en el entorno (Ruiz de la Rosa, García-Rodríguez y Delgado-Rodríguez, 2014). Así el entorno cambiante, de distinguido carácter tecnológico y de sostenibilidad, hace necesario el desarrollo de capacidades alineadas al contexto de las personas potencialmente emprendedoras. Es distinto en el caso de las personas estudiantes hombres y mujeres. A partir de esto, se establece la última de las hipótesis que relaciona las CE con la PA por sexos.

$H_{4}$ : Las Capacidades Emprendedoras (CE) en del alumnado universitario que influyen sobre la Propensión Actuar (PA) registran diferenciación de género.

Los trabajos que han abordado la realidad de la PA desde una perspectiva de género prueban la mayor presencia del sexo masculino respecto al sexo femenino en sus distintas fases (Gupta, Turban y Bhawe, 2008). En consecuencia, es importante favorecer la ruptura de los estereotipos emprendedores y, por tanto, respecto al propósito de emprender. Para ello, se puede fomentar un nuevo pensamiento que ejerza un mayor nivel de atracción sobre las mujeres alrededor de la PA (Zhang et al., 2014). Sin olvidar, los cambios en la posición actual de las mujeres en todos los ámbitos de la sociedad, las mujeres ahora tienen un entorno mucho más favorecedor para seguir su carrera profesional y tomar decisiones (Rózsa, Zbranková y Rahman, 2018).

\section{Método}

\section{Muestra y recogida de datos}

Esta investigación se apoya en una cuidada revisión de la literatura expuesta en el marco teórico y en una metodología descriptiva, inferencial y multivariada para contrastar las hipótesis planteadas. 
La población objeto de estudio queda definida como el alumnado matriculado en la asignatura de Políticas de Desarrollo Local de cuarto curso del Grado de Relaciones Laborales y Recursos Humanos de la Universidad de Sevilla entre 2012-13 a 2019-20, total de 2.127 personas.

El uso de la población estudiante universitaria viene avalado por distintos trabajos (Cui, Sun y Bell, 2019; García-Río, Baena-Luna, Sánchez-Torné y Pérez-Suárez, 2020). Tal y como señalan Harrison y List (2004), el colectivo de alumnado universitario es un grupo con un potencial emprendedor alto y, además, como grupo es representativo. El análisis de los propósitos emprendedores de la población universitaria favorece la comprensión sobre los elementos más relevantes que influyen en la intención emprendedora juvenil (Goyanes, 2015).

Tabla 2

Resultados descriptivos

\begin{tabular}{lcccccc}
\hline \multicolumn{1}{c}{$\begin{array}{c}\text { Variables } \\
\text { (variables independientes) }\end{array}$} & \multicolumn{2}{c}{ Total } & \multicolumn{2}{c}{ Hombre } & \multicolumn{2}{c}{ Mujer } \\
\hline & $\mathrm{N}$ & $\%$ & $\mathrm{~N}$ & $\%$ & $\mathrm{~N}$ & $\%$ \\
Género & 663 & $100 \%$ & 213 & $32,13 \%$ & 450 & $67,87 \%$ \\
Padre o madre con negocio & 212 & $31,97 \%$ & 74 & $34,74 \%$ & 138 & $30,67 \%$ \\
Otra persona con negocio & 470 & $70,88 \%$ & 154 & $72,30 \%$ & 316 & $70,22 \%$ \\
Le gustaría tener su propia empresa & 493 & $74,35 \%$ & 172 & $80,75 \% *$ & 321 & $71,33 \% *$ \\
Tiene una idea & 340 & $51,28 \%$ & 120 & $56,34 \%$ & 220 & $48,89 \%$ \\
${ }^{*} \mathrm{p}<0,05$ & & & & & & \\
\hline
\end{tabular}

Fuente: Elaboración propia a partir de resultados de la encuesta.

Para recoger la percepción sobre la actitud emprendedora del alumnado universitario se empleó una encuesta ad hoc en el mes de noviembre de cada curso académico, suministrada por correo. Con el fin de velar por la veracidad de las respuestas la encuesta fue respondida de forma voluntaria y se garantizó el anonimato de la muestra participante al no requerir los datos personales. Además, el proceso fue seguido y vigilado para que una misma persona no respondiera dos veces.

Se procedió a un muestreo no probabilístico incidental, donde se suministró la encuesta al total de personas matriculada, y se obtuvo un total de 663 respuestas. A pesar de los inconvenientes de este método para generalizar las conclusiones, se destaca que el volumen de respuestas implica un reducido margen de error del 3,2\% al $95 \%$ de confianza. Además, tanto el porcentaje obtenido por sexo (hombres $67,87 \%$ y mujeres $32,13 \%$ ) como por edad (71,50\% entre $21-25$ años) de la muestra se aproximan a los observados en la población. Así pues, se puede indicar que los resultados de esta investigación son válidos para establecer conclusiones sobre la población estudiantil del Grado de Relaciones Laborales y Recursos Humanos de la Universidad de Sevilla. 
Los resultados descriptivos (Tabla 2) evidencian unos datos muy similares entre ambos sexos, excepto en la deseabilidad de tener su propia empresa donde ellos muestran un porcentaje significativamente superior.

\section{Instrumento}

El instrumento para la medición de la PA fueron las cuestiones incluidas en la encuesta planteada por Liñán y Chen (2009) sobre la modelización de las variables adecuadas para medir la PA. Dichas cuestiones pueden agruparse e identificar las variables del modelo de Shapero y Sokol (1982) tal como muestra la Tabla 3.

Esta se compone por cuestiones de registro (género, edad, municipio de residencia), que anteceden a 4 cuestiones dicotómicas (Sí/No) para determinar si el alumnado ha considerado crear una empresa y si tiene modelos de referencia emprendedora. En último lugar, se hallan dos bloques compuestos por 22 afirmaciones sobre PA, CP, PV y Capacidades emprendedoras valoradas por una Escala Likert de cinco posiciones. Donde el alumnado debe indicar su grado de alineación con las afirmaciones según las posiciones que van de 1 (totalmente desacuerdo) a 5 (totalmente de acuerdo). Las cuestiones que componen estos bloques se pueden ver en la Tabla 3. Las medias de los dos primeros se trabajaron de manera agregada ya que las cuestiones que las componen miden el bloque en su conjunto.

Las cuestiones tratadas fueron contrastadas con otras investigaciones relacionadas como son las de: Liñán, Rodríguez-Cohard y Rueda-Cantuche (2011); Liñán, Urbano y Guerrero (2011); Srivastava y Misra (2017), Sánchez-Torné y Pérez-Suárez (2019), entre otros.

Tabla 3

Preguntas que componen las variables de $P A, C P, P V$ y $C E$

\section{Propensión a Actuar (PA) (variable dependiente)}

Estoy preparado/a para hacer lo necesario para convertirme en una persona emprendedora

Mi meta profesional es llegar a ser una persona emprendedora

Realizaré todos los esfuerzos precisos para iniciar y continuar mi propia empresa

Estoy decidido/ a crear una empresa en el futuro

Pienso con bastante firmeza en crear mi propia empresa

Conveniencia Percibida (CP) (variable independiente)

Ser una persona emprendedora tiene más ventajas que inconvenientes

Ser una persona emprendedora me resulta atractivo

Si tuviese la oportunidad y los medios me gustaría crear mi propia empresa 


\section{Propensión a Actuar (PA) (variable dependiente)}

Convertirme en persona emprendedora sería algo que me dejaría muy satisfecho

De entre varias opciones, preferiría ser persona emprendedora

Percepción de Viabilidad (PV) (variable independiente)

Crear una empresa y mantenerla en funcionamiento sería fácil para

Estoy capacitado/a para poner en marcha una empresa viable

Puedo controlar el proceso de creación de una nueva empresa

Conozco los detalles prácticos necesarios para crear una empresa

Sé cómo desarrollar un proyecto empresarial

Si intentase crear una empresa, tendría muchas posibilidades de éxito

Capacidades Emprendedoras (CE) (variable independiente)

Soy perfectamente capaz de reconocer las oportunidades

Soy muy creativo/a

Soy capaz de resolver prácticamente cualquier problema que se me presente

Soy capaz de dirigir a otras personas y de comunicarles adecuadamente mis ideas e intenciones

Soy perfectamente capaz de desarrollar nuevos productos y servicios

Soy perfectamente capaz de establecer una red de contactos personales y profesionales

Fuente: Elaboración propia a partir del cuestionario de Liñán y Chen (2009).

Así mismo, la escala utilizada ha sido validada con estudios predecesores y ha presentado gran consistencia, pues los resultados del Alpha de Cronbach por bloques de preguntas (Tabla 4) están por encima de 0,7. Este valor es considerado como aceptable (George y Mallery, 2003).

Tabla 4

Prueba Alpha de Cronbach. Fiabilidad de la Escala

\begin{tabular}{lc}
\hline Bloques de preguntas & Alpha de Cronbach \\
\hline CP & 0,853 \\
PV & 0,854 \\
PA & 0,919 \\
\hline
\end{tabular}

Fuente: Elaboración propia a partir de resultados de la encuesta. 


\section{Procedimiento análisis de datos}

Las pruebas estadísticas son las correspondientes para contrastar las hipótesis plantadas en esta investigación. Para ello se empleó un modelo regresión lineal múltiple tras un análisis de inferencia que determinó si ambos sexos presentaban circunstancias y valoraciones diferentes ante las variables.

El análisis de inferencia para detectar las posibles diferencias de valoración en las respuestas de alumnas y alumnos, con un nivel de confianza del $95 \%$ y el error del $5 \%$, no tuvo en cuenta las respuestas Ns/Nc:

1) Prueba $Z$ para contrastar proporciones. Es empleada en las cuestiones con respuesta dicotómica (modelos de referencia y considerar crear un negocio).

2) Prueba $U$ de Mann-Whitney para diferir medias poblacionales (prueba no paramétrica, debido a que los resultados no siguen una distribución normal).

Para medir la influencia de cada variable en la PA se desarrolló un modelo regresión lineal múltiple. Con esta herramienta se pudo verificar qué variable afecta a la PA del conjunto de la muestra y por sexos.

El modelo de regresión lineal múltiple sigue la expresión matemática (Lind, Marchal y Wathen, 2005) y se ajusta con nuestro modelo PA de la siguiente forma:

$\mathrm{Y}=\mathrm{a} 0+\beta 1 \mathrm{X} 1+\beta 2 \mathrm{X} 2+\beta 3 \mathrm{X} 3+\ldots+\beta \mathrm{nXn}+\mathrm{e}$

$\mathrm{Y}=$ variable dependiente. En este caso se estudia la PA.

$\beta 1, \beta 2, \beta 3 \ldots \beta \mathrm{n}=$ coeficiente (peso) de la variable independente.

$\mathrm{X} 1, \mathrm{X} 2, \mathrm{X} 3 \ldots \mathrm{Xn}$ = variables independientes (en nuestro modelo: el sexo, persona progenitora $\mathrm{u}$ otra persona emprendedora, me gustaría tener mi propia empresa, tener una idea de negocio, los constructos CP, PV y CE. Esta última de forma individualizada).

$\mathrm{e}=$ residuo. Este modelo comprueba que son residuos homocedásticos por lo que tiende a ser cero siendo no relevante para el análisis.

Los análisis de regresión son de uso vigente por las personas investigadoras de diferentes materias, en particular, sobre el tema objeto de análisis en esta investigación destacan los estudios de Joensuu-Salo, Varamäki y Viljamaa (2015); Jemari et al. (2017); Barba-Sánchez y Atienza-Sahuquillo (2018).

\section{Resultados}

En relación con la PA, se descubre un porcentaje significativamente superior en los hombres en tres variables observadas (Tabla 5): PV, CP y CE. Además, y de forma individualizada, en los hombres destacan: las capacidades de reconocer oportunidades, creatividad, resolver problemas y establecer una red de contactos. 
Tabla 5

Elementos de la PA

\begin{tabular}{|c|c|c|c|c|c|c|}
\hline \multirow[t]{2}{*}{ Variables } & \multicolumn{2}{|c|}{ Total } & \multicolumn{2}{|c|}{ Hombre } & \multicolumn{2}{|c|}{ Mujer } \\
\hline & Media & DV & Media & DV & Media & $\mathrm{DV}$ \\
\hline PA & 2,89 & 0,99 & $3,14^{*}$ & 1,00 & $2,76^{*}$ & 0,96 \\
\hline $\mathrm{CP}$ & 3,63 & 0,85 & $3,80^{*}$ & 0,84 & $3,55^{*}$ & 0,85 \\
\hline PV & 2,74 & 0,73 & $2,92^{*}$ & 0,71 & $2,66^{*}$ & 0,72 \\
\hline CE & 3,35 & 0,69 & $3,50^{*}$ & 0,70 & $3,28^{*}$ & 0,68 \\
\hline \multicolumn{7}{|l|}{ Capacidades Emprendedoras detalladas } \\
\hline Reconocer las oportunidades & 3,25 & 0,93 & $3,40^{*}$ & 0,99 & $3,18^{*}$ & 0,90 \\
\hline Creativa & 3,26 & 1,06 & $3,44^{*}$ & 0,97 & $3,17^{*}$ & 1,08 \\
\hline $\begin{array}{l}\text { Resolver prácticamente cualquier } \\
\text { problema que se me presente }\end{array}$ & 3,41 & 0,86 & $3,54^{*}$ & 0,85 & $3,35^{*}$ & 0,85 \\
\hline $\begin{array}{l}\text { Dirigir a otras personas y de co- } \\
\text { municarles adecuadamente mis } \\
\text { ideas e intenciones }\end{array}$ & 3,68 & 0,88 & 3,79 & 0,87 & 3,62 & 0,88 \\
\hline $\begin{array}{l}\text { Desarrollar nuevos productos y } \\
\text { servicios }\end{array}$ & 3,14 & 0,89 & 3,26 & 0,91 & 3,08 & 0,88 \\
\hline $\begin{array}{l}\text { Establecer una red de contactos } \\
\text { personales y profesionales }\end{array}$ & 3,38 & 0,94 & $3,55^{*}$ & 0,91 & $3,29 *$ & 0,94 \\
\hline${ }^{*} \mathrm{p}<0,05$ & & & & & & \\
\hline
\end{tabular}

Fuente: Elaboración propia a partir de resultados de la encuesta.

A continuación, se presenta el modelo de PA propuesto para el total del alumnado por género (Tabla 6). Este ha sido elaborado a partir de un análisis de regresión lineal múltiple. En él se presentan todas las variables independientes, pero solo aquellas marcadas con asterisco (de uno a tres dependiendo de su nivel de significatividad) presentan incidencia en la PA.

Las siete variables independientes que influyen positivamente en la PA del conjunto estudiantil son: tener una persona progenitora emprendedora, gustarle tener su propia empresa, tener una idea de negocio, la CP, la PV y la capacidad de reconocer oportunidades y desarrollar nuevos productos y servicios. Por otro lado, dos variables se presentan con incidencia negativa: el género (ser mujer) y la capacidad de resolver cualquier problema.

En el modelo de PA para los alumnos, las cinco variables independientes con influencia positiva que resultaron ser significativas fueron: persona progenitora emprendedora, tener una idea de negocio, la CP, la PV y establecer una red de contactos profesionales y personales. 
En el modelo de PA para las alumnas, se dispone de siete variables independientes con influencia positiva: persona progenitora emprendedora, gustarle tener una empresa, tener una idea de negocio, la CP, la PV, reconocer oportunidades y capacidad de desarrollar nuevos productos y servicios.

Tabla 6

Modelo explicativo de las variables que influyen en la PA del alumnado

\begin{tabular}{|c|c|c|c|}
\hline & $\begin{array}{l}\text { Modelo total del } \\
\text { alumnado }\end{array}$ & $\begin{array}{l}\text { Modelo } \\
\text { alumnos }\end{array}$ & $\begin{array}{l}\text { Modelo } \\
\text { alumnas }\end{array}$ \\
\hline Constante. & $-0,448^{* *}$ & $-0,793^{* *}$ & $-0,396^{*}$ \\
\hline Sexo. & $-0,114^{*}$ & & \\
\hline $\begin{array}{l}\text { Padre o madre con negocio (persona pro- } \\
\text { genitora emprendedora / modelo de refer- } \\
\text { encia). }\end{array}$ & $0,165^{* *}$ & $0,204^{*}$ & $0,162^{*}$ \\
\hline $\begin{array}{l}\text { Otra persona con negocio (modelo de ref- } \\
\text { erencia). }\end{array}$ & $-0,116$ & $-0,028$ & $-0,133$ \\
\hline Le gustaría tener su propia empresa. & $0,396^{* * *}$ & 0,221 & $0,454^{* * *}$ \\
\hline Idea de negocio. & $0,210^{* * *}$ & $0,272^{* *}$ & $0,192^{* *}$ \\
\hline $\mathrm{CP}$ & $0,518^{* * *}$ & $0,628^{* * *}$ & $0,476^{* * *}$ \\
\hline PV & $0,200^{* * *}$ & $0,178^{*}$ & $0,210^{* * *}$ \\
\hline Reconocer las oportunidades. & $0,102^{* *}$ & 0,057 & $0,114^{* *}$ \\
\hline Creatividad. & 0,006 & 0,035 & $-0,005$ \\
\hline $\begin{array}{l}\text { Resolver prácticamente cualquier prob- } \\
\text { lema que se me presente. }\end{array}$ & $-0,083^{*}$ & $-0,140$ & $-0,074$ \\
\hline $\begin{array}{l}\text { Dirigir a otras personas y de comunicarles } \\
\text { adecuadamente mis ideas e intenciones. }\end{array}$ & $-0,019$ & 0,029 & $-0,036$ \\
\hline Desarrollar nuevos productos y servicios. & $0,140^{* * *}$ & 0,094 & $0,177^{* * *}$ \\
\hline $\begin{array}{l}\text { Establecer una red de contactos person- } \\
\text { ales y profesionales. }\end{array}$ & 0,057 & $0,124^{*}$ & 0,013 \\
\hline $\mathrm{R}$ & 0,821 & 0,829 & 0,816 \\
\hline $\mathrm{R}^{2}$ & 0,675 & 0,688 & 0,666 \\
\hline $\mathrm{R}^{2}$ ajustado & 0,667 & 0,664 & 0,655 \\
\hline Error estándar de la estimación & 0,559 & 0,560 & 0,559 \\
\hline Sig. Test ANOVA & 0,000 & 0,000 & 0,000 \\
\hline${ }^{*} \mathrm{p}<0.05 ;{ }^{* *} \mathrm{p}<0.01 ;{ }^{* * *} \mathrm{p}<0.001$ & & & \\
\hline
\end{tabular}

Fuente: Elaboración propia a partir de resultados de la encuesta. 
Para determinar la bondad de ajuste del modelo y su grado de fiabilidad se ha recurrido al análisis del $\mathrm{R}^{2}$ y ANOVA. En la Tabla 6, se puede comprobar como el $\mathrm{R}^{2}$ exhibe unos valores aceptables. Más si cabe teniendo en cuenta que se trata de la rama de ciencias sociales, por lo que el modelo se ajusta debidamente. Además, el test ANOVA fue significativo para los tres modelos, siendo por tanto su predicción adecuada y resultando un modelo lineal apropiado.

\section{Discusión y Conclusiones}

El objetivo de la investigación se logra al determinar varios elementos significativos que afectan a la PA del alumnado universitario desde una perspectiva de género. En consecuencia, se comprueban unas diferencias significativas entre alumnas y alumnos en la PA y las variables que inciden en la PA. El conjunto de alumnado presentó un modelo de PA definido por la influencia positiva de siete variables: tener una persona progenitora emprendedora, experimentar tener una empresa, tener una idea de negocio, la CP, la PV, y reconocer las oportunidades y desarrollar nuevos productos y servicios. Así mismo, este modelo de PA presenta una variable que reduce la probabilidad de incrementar la PA que fue el género (ser mujer reduce la PA).

En el caso del grupo de hombres, muestra niveles superiores respecto a las mujeres en ocho elementos: PA, PV, CP, le gustaría tener su propia empresa, reconoce las oportunidades, creatividad, resolver problemas y establecer una red de contactos.

No obstante, hay que matizar que el alumnado objeto de estudio no corresponde a un curso de educación emprendedora, por lo que las diferencias halladas no se refieren a la cualificación. Si bien la educación emprendedora no supone necesariamente un efecto positivo en la PA del alumnado, sí incide en la valoración sobre los conocimientos necesarios para crear una empresa (Sánchez-Torné y Pérez-Suárez, 2019). Por otro lado, las posibles anomalías quedarían disueltas ya que la información recopilada comprende seis cursos académicos. Al mismo tiempo, la población muestra unas valoraciones dispares en perspectiva de género, pues ellos presentan un resultado superior en la mayoría de las cuestiones formuladas.

Con el fin de obtener una información definida sobre estas diferencias, se llevó a cabo un modelo de regresión lineal. A partir de él, se obtiene que a los alumnos solo le influyen cinco variables a la hora de considerar crear una empresa mientras que a las mujeres le influyen siete (Tabla 7). Para ambos sexos se sustenta la Hipótesis 1 (CP influye en la PA) e Hipótesis 2 (PV influye en la PC). Así, se constata que la CP y la PV son predictores del PA tanto para hombres como para mujeres, por lo que se coincide con las averiguaciones de otros trabajos (Autio et al., 2001; Ali, Lu y Wang, 2012; Zhang et al., 2014). Cuando los modelos de referencia emprendedora son las personas progenitoras, esta variable sí influye en la PA del alumnado femenino y masculino. En este caso no se sustenta la Hipótesis 3 (los modelos de referencia que tienen una relación positiva con la PA es distinta entre alumnas y alumnos) puesto que la influencia es igual para ambos sexos. Esto revela la importancia de incluir los modelos de referencia emprendedora y fomentar la creatividad (generación de ideas) en la docencia, como elemento potenciador de la PA. 
En relación con las $\mathrm{CE}$, solo los hombres registran la capacidad de establecer una red de contactos profesionales y personales. Mientras que, las mujeres registran las capacidades de reconocer oportunidades y desarrollar nuevos productos y servicios. De ahí que, la Hipótesis 4 se sustente (las capacidades emprendedoras del alumnado universitario que influyen sobre la PA registran diferenciación de género) y evidencie la necesidad de regenerar el desarrollo de las CE paritarias en el aula, como elemento potenciador de la PA.

Tabla 7

Resumen de las variables que influyen a la hora de considerar crear una empresa para las alumnas y los alumnos

\begin{tabular}{ccc}
\hline Variables & Alumnas & Alumnos \\
\hline $\begin{array}{c}\text { Modelos de referencia em- } \\
\text { prendedora (MRE): madre/ } \\
\text { padre u otra persona. } \\
\text { Le gustaría tener su propia } \\
\text { empresa. }\end{array}$ & $\begin{array}{c}\text { Influencia positiva: Madre o } \\
\text { Padre emprendedor. }\end{array}$ & $\begin{array}{c}\text { Influencia positiva: Madre o } \\
\text { Padre emprendedor. }\end{array}$ \\
Idea de negocio. & Influencia positiva. & Ninguna influencia. \\
Conveniencia Percibida. & Influencia positiva. & Influencia positiva. \\
Percepción de Viabilidad. & Influencia positiva. & $\begin{array}{c}\text { Influencia positiva. } \\
\text { Influencia positiva. }\end{array}$ \\
Capacidades Emprendedoras. & $\begin{array}{c}\text { Influencia positiva: Reconocer positiva. } \\
\text { oportunidades, capacidad de } \\
\text { desarrollar nuevos productos } \\
\text { y servicios. }\end{array}$ & $\begin{array}{c}\text { Influencia positiva: Establ- } \\
\text { ecer una red de contactos } \\
\text { profesionales y personales. }\end{array}$ \\
\hline
\end{tabular}

Fuente: Elaboración propia a partir de resultados de la encuesta.

Las investigaciones sobre la capacitación indican que la educación emprendedora puede salvar la brecha de género, aunque existen pocas investigaciones empíricas que evalúen la validez y el impacto de las iniciativas. Esto muestra una naciente oportunidad de exploración académica (Elert, Sjöö y Wennberg, 2020).

En definitiva, las implicaciones de la investigación pasan por sumar a la representación del género desde la demostración empírica que las mujeres distinguen más elementos de la PA que los hombres, aunque también constata que los hombres exhiben una mayor PA que las mujeres (Babikova y Bucek, 2019; Cera et al., 2020). No obstante, en la población universitaria se observa una respuesta similar entre hombres y mujeres en relación a la PA. Por tanto, la PA universitaria registra una leve brecha de género (2 de 4 variables de la Tabla 7).

Las limitaciones de la investigación refieren a la naturaleza de estudiar un micromundo, como fue el alumnado del Grado de Relaciones Laborales y Recursos Humanos de la Universidad de Sevilla y con ello, la acotación geográfica, temporal y económica. Además, el método de captación de datos a través de una encuesta se basa en una autoevaluación, 
por lo que es parcialmente información sesgada. Este estudio no puede representar una imagen exacta de la PA universitaria del territorio, pero sí aportar evidencias de una muestra representativa del mencionado grado. Con ello sumar resultados a la literatura sobre la educación emprendedora.

En futuras líneas de investigación se requiere un análisis longitudinal amplio para pormenorizar la relación de la PA por género con los MRE y la educación empresarial. Así como la combinación con metodologías complementarias a la encuesta para mitigar los posibles componentes subjetivos. Las derivaciones prueban que hay que avanzar en la investigación sobre la PA y el Género, siendo el género una variable significativa para planificar e implementar los programas superiores de educación empresarial.

\section{Referencias}

Ajzen, I. (1991). The theory of planned behavior. Organizational behavior and human decision processes, 50, 179-211. Doi: https://doi.org/10.1016/0749-5978(91)90020-T

Ali, S., Lu, W. \& Wang, W. (2012). Determinants of entrepreneurial intentions among the college students in: China and Pakistan. Journal of Education and Practice, 3(11), 13-22. Recuperado de https://preview.tinyurl.com/y4alh2d5

Asghar, M. Z., Gul, F., Seitamaa-Hakkarainen, P. \& Tasdemir, M. Z. (2019). Validating Entrepreneurial Intentions Questionnaire to Assess the Impact of Entrepreneurship Education. Education and Science, 44(197), 383-399. Doi: https://doi.org/10.15390/ EB.2019.6105

Autio, E., Keeley, R., Klofsten, M., Parker, G. G. \& Hay, M. (2001). Entrepreneurial Intent among Students in Scandinavia and in the USA. Enterprise and Innovation Management Studies, 2(2), 145-160. Doi: https://doi.org/10.1080/14632440110094632

Babikova, K., \& Bucek, J. (2019). A Model Replication with an Extension of Students' Perception of Prospective Employer Attractiveness. Journal of Competitiveness, 11(2), 5-21. https://doi.org/10.7441/joc.2019.02.01

Baena-Luna, P., García-Río, E. \& Monge-Agüero, M. (2020). Entrecomp: marco competencial para el emprendimiento. Una revisión sistemática de la literatura sobre su uso y aplicación. Información Tecnológica, 31(2), 163-172. Doi: http://dx.doi.org/10.4067/ S0718-07642020000200163

Barba-Sánchez, V. \& Atienza-Sahuquillo, C. (2018). Entrepreneurial intention among engineering students: The role of entrepreneurship education. European Research on Management and Business Economics, 24(1), 53-61. Doi: https://doi.org/10.1016/j. iedeen.2017.04.001

Cera, G., Mlouk, A., Cera, E. \& Shumeli, A. (2020). The Impact of Entrepreneurship Education on Entrepreneurial Intention. A Quasi-Experimental Research Design. Journal of Competitiveness, 12(1), 39- 56. Doi: https://doi.org/10.7441/joc.2020.01.03

Cui, J., Sun, J. \& Bell, R. (2019). The impact of entrepreneurship education on the entrepreneurial mindset of college students in China: The mediating role of inspiration and the role of educational attributes. The International Journal of Management Education. (Article in press) Doi: https://doi.org/10.1016/j.ijme.2019.04.001

Elert, N., Sjöö, K., \& Wennberg, K. (2020). When Less Is More: Why Limited Entrepreneurship Education May Result in Better Entrepreneurial Outcomes. Research 
Institute of Industrial Economics, 1322, 1-43. Available in https:/www.ifn.se/wfiles/ wp/wp1322.pdf

Garba, A.S., Kabir, S. \& Nalado, A.M. (2014). An Assessment of Students Entrepreneurial Intentions in Tertiary Institution: A Case of Kano State Polytechnic, Nigeria. International Journal of Asian Social Science, 4(3), 434-443. Recuperado de https:// preview.tinyurl.com/y49fuk7j

García-Río, E., Baena-Luna, P., Sánchez-Torné, I., \& Pérez-Suárez, M. (2020). Entrepreneurial Intentions determinants elements in university students. A study from a Gender Perspective. 3C Empresa. Investigación y pensamiento crítico, 41(9), 89-107. http://doi.org/10.17993/3cemp.2020.090141.89-107

George, D. y Mallery, M. (2003). Using SPSS for Windows step by step: a simple guide and reference. Boston, MA: Allyn y Bacon.

Gielnik, M. M., Uy, M. A., Funken, R., \& Bischoff, K. M. (2017). Boosting and sustaining passion: A long-term perspective on the effects of entrepreneurship training. Journal of Business Venturing, 32(3), 334-353. Doi: https://doi.org/10.1016/j.jbusvent.2017.02.003

González-Serrano, M. H., Valantine, I., Pérez, C., Aguado, S., Calabuig, F. \& Crespo, J. J. (2016). La influencia del género y de la formación académica en la intención de emprender de los estudiantes de ciencias de la actividad física y el deporte. Intangible Capital, 12(3), 759-788. Doi: http://dx.doi.org/10.3926/ic.783

Goyanes, M. (2015). Apoyo estructural en la intención emprendedora de estudiantes de periodismo y comunicación audiovisual en España. El Profesional de La Información, 24(1), 55-61. Doi: https://doi.org/10.3145/epi.2015.ene.07

Gupta, V. K., Turban, D. B. \& Bhawe, N. M. (2008). The effect of gender stereotype activation on entrepreneurial intentions. Journal of Applied Psychology, 93(5), 10531061. Doi: https://doi.org/10.1037/0021-9010.93.5.1053

Gupta, V. K., Wieland, A. M., \& Turban, D. B. (2019). Gender Characterizations in Entrepreneurship: A Multi-Level Investigation of Sex-Role Stereotypes about HighGrowth, Commercial, and Social Entrepreneurs. Journal of Small Business Management, 57(1), 131-153. Doi: https://doi.org/10.1111/jsbm.12495

Harrison, G. W. \& List, J. A. (2004). Field Experiments. Journal of Economic Literature, 42(4), 1009-1055. Doi: https://doi.org/10.1257/0022051043004577

Jemari, M. A., Kasuma, J., Kamaruddin, H. M., Tama, H. A., Morshidi, I. \& Suria, K. (2017). Relationship between human capital and social capital towards social entrepreneurial intention among the public university students. International Journal of Advanced and Applied Sciences, 4(12), 179-184. Doi: https://doi.org/10.21833/ ijaas.2017.012.032

Joensuu-Salo, S., Varamäki, E. \& Viljamaa, A. (2015). Beyond intentions-what makes a student start a firm? Education+Training, 57(8/9), 853-873. Doi: https://doi.org/10.1108/ ET-11-2014-0142

Karimi, S., Biemans, H. J. A., Lans, T., Mulder, M. \& Chizari, M. (2012). The Impact of Entrepreneurship Education on Students Entrepreneurial Intentions and Opportunity Identification Perceptions. En Proceedings of 26th Annual RENT Conference. Lyon. Doi: https://doi.org/10.2139/ssrn.2275638 
Klapper, R. \& Léger-Jarniou, C. (2006). Entrepreneurship Intention among French Grande ÉCole and University Students: An Application of Shapero's Model. Industry and Higher Education, 20(2), 97-110. Doi: https://doi.org/10.5367/000000006777699900

Krueger Jr., N.F. \& Brazeal, D.V. (1994). Entrepreneurial potential and potential entrepreneurs, Entrepreneurship Theory and Practice, 18(3), 91-104. Doi: https://doi. org/10.1177/104225879401800307

Kuehn, K.W. (2008). Entrepreneurial intentions research: Implications for Entrepreneurship education. Journal of Entrepreneurship Education, 11, 87-98. Recuperado de https://preview.tinyurl.com/yyvb8zob

Lim, S. \& Envick, B. R. (2013). Gender and entrepreneurial orientation: A multi-country study. International Entrepreneurship and Management Journal, 9(3), 465-482. Doi: https://doi.org/10.1007/s11365-011-0183-2

Liñán, F. \& Chen, W. (2009). Development and Cross-Cultural application of a specific instrument to measure entrepreneurial intentions. Entrepreneurship theory and practice, 33(3), 593-617. Doi: https://doi.org/10.1111/j.1540-6520.2009.00318.x

Liñán, F., Rodríguez-Cohard, J. C. \& Rueda-Cantuche, J. M. (2011). Factors affecting entrepreneurial intention levels: a role for education. International entrepreneurship and management Journal, 7(2), 195-218. Doi: https://doi.org/10.1007/s11365-010-0154-z

Liñán, F., Urbano, D. \& Guerrero, M. (2011). Regional variations in entrepreneurial cognitions: start-up intentions of university students in Spain. Entrepreneurship and Regional Development, 23(3-4), 187-215. Doi: https://doi.org/10.1080/08985620903233929

Lind, D. A., Marchal, W. G. \& Wathen, S. A. (2005). Estadística aplicada a los negocios y la economía. McGraw-Hill.

Maresch, D., Harms, R., Kailer, N. \& Wimmer-Wurm, B. (2016). The impact of Entrepreneurship education on the entrepreneurial intention of students in science and engineering versus business studies university programs. Technological Forecasting and Social Change, 104, 172-179. Doi: https://doi.org/10.1016/j.techfore.2015.11.006

Martínez-García, I.; Padilla-Carmona, M.T. \& Suárez-Ortega, M. (2019). Aplicación de la metodología Delphi a la identificación de factores de éxito en el emprendimiento. Revista de Investigación Educativa, 37(1), 129-146. Doi: http://dx.doi.org/10.6018/ rie.37.1.32091

Medina, E. G., Moncayo, O. F., Jácome, L. F. \& Albarrasin, M. V. (2017). El emprendimiento en el sistema universitario. Revista Didascálica: DyE, VIII (1), 163-178. Recuperado de https://dialnet.unirioja.es/servlet/articulo?codigo $=6630552$

Naval-Castelao, M. O., González-Pascual, J. L., Jordán-Ramos, A. \& Ruiz-Pomada, A. (2015). Universidad y emprendimiento. Intención emprendedora en estudiantes de universidades privadas madrileñas. Revista de Investigación En Educación, 13(2), 187-205. Recuperado de http://reined.webs.uvigo.es/index.php/reined/article/view/229

Neira, I., Portela, M., Fernández, L. \& Rodeiro, D. (2013). Una actitud emprendedora: Un enfoque a través de los jóvenes y el género. En A. López-Díaz (Ed.), Emprender una perspectiva de género (pp. 69-84). Servicios de Publicaciones de la Universidad de A Coruña. Recuperado de https://preview.tinyurl.com/y36zbten

Premand, P., Brodmann, S., Almeida, R., Grun, R. \& Barouni, M. (2016). Entrepreneurship Education and Entry into Self-Employment Among University Graduates. World Development, 77, 311-327. Doi: https://doi.org/10.1016/j.worlddev.2015.08.028 
Romero-Martínez, A.M. \& Milone, M. (2016). El emprendimiento en España: Intención emprendedora, motivaciones y obstáculos. Journal Globalization, Competitiveness and Governability, 10(1), 95-109. Doi: https://doi.org/10.3232/GCG.2016.V10.N1.05

Rózsa, Z., Zbranková, H. \& Rahman, A. (2018). Gender differences of managing banks' credit portfolio. Polish Journal of Management Studies, 18(2), 222-283. https://doi. org/10.17512/pjms.2018.18.2.22

Ruiz de la Rosa, C., García-Rodríguez, F. \& Delgado-Rodríguez, N. (2014). Condicionantes de la intención emprendedora en el alumnado universitario: Un análisis desde la perspectiva de género. Revista de Estudios Empresariales. Segunda Época, 2(2), 81-96. Doi: https://doi.org/10.17561//ree.v2019n2

Sánchez-Cañizares, S. M. \& Fuentes-García, F. J. (2013). Mujer y emprendimiento: Un análisis en el contexto universitario español. Revista de Ciencias Sociales, 19(1), 140-153. Doi: https://doi.org/10.31876/rcs.v19i1.25612

Sánchez-García, J. C. (2010): Evaluación de la personalidad emprendedora: validez factorial del cuestionario de orientación emprendedora (COE). Revista Latinoamericana de Psicología, 42(1), 41-52.

Sánchez-Torné, I. \& Pérez-Suárez, M. (2019). El impacto de la educación en la intención emprendedora de los estudiantes del Grado de Economía. Revista de Estudios Empresariales. Segunda Época, 1(1), 22-40. Doi: https://doi.org/10.17561/ree.v2019n1.2

Schmitz, A., Urbano, D., Dandolini, G. A., de Souza, J. A. \& Guerrero, M. (2017). Innovation and Entrepreneurship in the academic setting: a systematic literature review. International Entrepreneurship and Management Journal, 13(2), 369-395. Doi: https://doi. org/10.1007/s11365-016-0401-z

Shapero, A. \& Sokol, L. (1982): The social dimensions of entrepreneurship. Encyclopedia of Entrepreneurship, 72-90. Recuperado de https://ssrn.com/abstract=1497759

Srivastava, S. \& Misra, R. (2017). Exploring antecedents of entrepreneurial intentions of young women in India: A multi-method analysis. Journal of Entrepreneurship in Emerging Economies, 9(2), 181-206. Doi: https://doi.org/10.1108/JEEE-04-2016-0012

Startiene, G., \& Remeikiene, R. (2008). Gender gap in entrepreneurship. Engineering Economics, 5(60), 95-103. Doi: https://doi.org/10.2139/ssrn.3282574

Zambrano-Vargas, S. M., \& Vázquez-García, A. W. (2019). Algunas perspectivas teóricas para el estudio del emprendimiento y el género. Saber, Ciencia y Libertad, 14(1), 159-170. Doi: https://doi.org/10.18041/2382-3240/saber.2019v14n1.5216

Zhang y., Duysters, G. \& Cloodt, M. (2014). The role of Entrepreneurship education as a predictor of university students' entrepreneurial intention. International Entrepreneurship and Management Journal, 10(3), 623-641. Doi: https://doi.org/10.1007/s11365-012-0246-z

Zhang, H. (2017). Complexity absorption: A processual strategic approach to corporate entrepreneurship strategy. Frontiers of Business Research in China, 11(1), 1-20. Doi: https://doi.org/10.1186/s11782-017-0015-5

Fecha de recepción: 18 de mayo de 2020.

Fecha de revisión: 12 de junio de 2020.

Fecha de aceptación: 13 de noviembre de 2020. 